\title{
A Direct Comparison of Inverted and Non-inverted Growths of GalnP Solar Cells
}

Myles Steiner, John Geisz, Robert Reedy Jr., Sarah Kurtz

33 ${ }^{\text {rd }}$ IEEE Photovoltaic Specialists Conference San Diego, CA May 13, 2008 


\section{Motivation}

Growing inverted cells may enable technological advances in solar cell fabrication, leading to higher efficiencies.

Differences in dopant diffusion during inverted vs. upright growths may lead to:

$\rightarrow$ Differences in atomic depth profiles

$\rightarrow$ Changes in carrier concentrations

$\rightarrow$ Higher contact resistance

$\rightarrow$ Lower overall performance 


\section{Inverted triple junction}

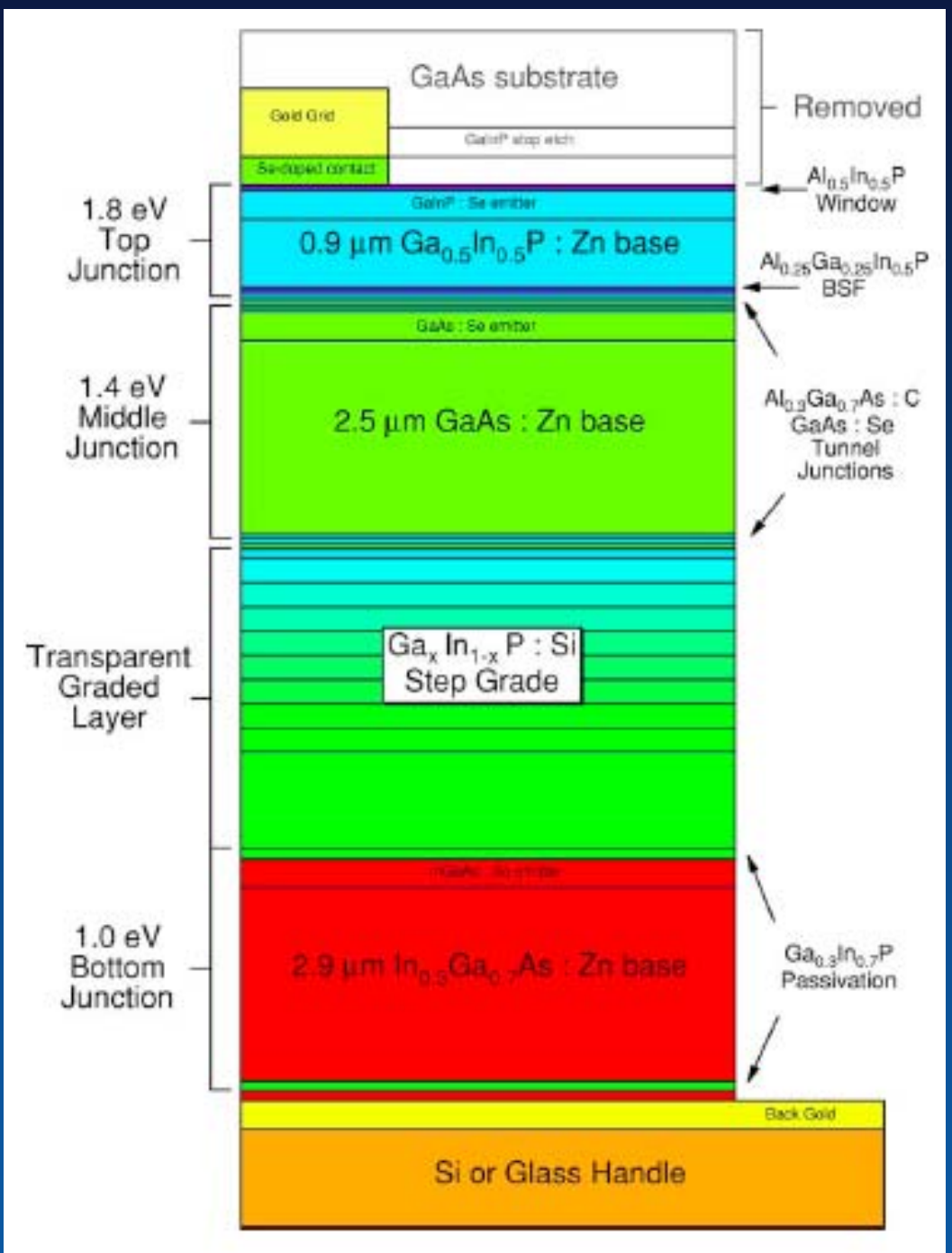

Geisz et al., 33 ${ }^{\text {rd }}$ PVSC (2008)

1.0-eV lattice-mismatched InGaAs bottom subcell enables a higher total efficiency: $39.2 \%$ @131 suns.

Growing the bottom subcell last avoids threading the middle and top subcells with dislocations.

Geisz et al., APL 91, 023502 (2007) 


\title{
This talk...
}

Solar cell characteristics

SIMS depth profiles analysis

\author{
Top contact resistance
}

Selenium diffusion in GalnAsN 


\section{Layer structures}

\section{Upright growth Inverted growth}

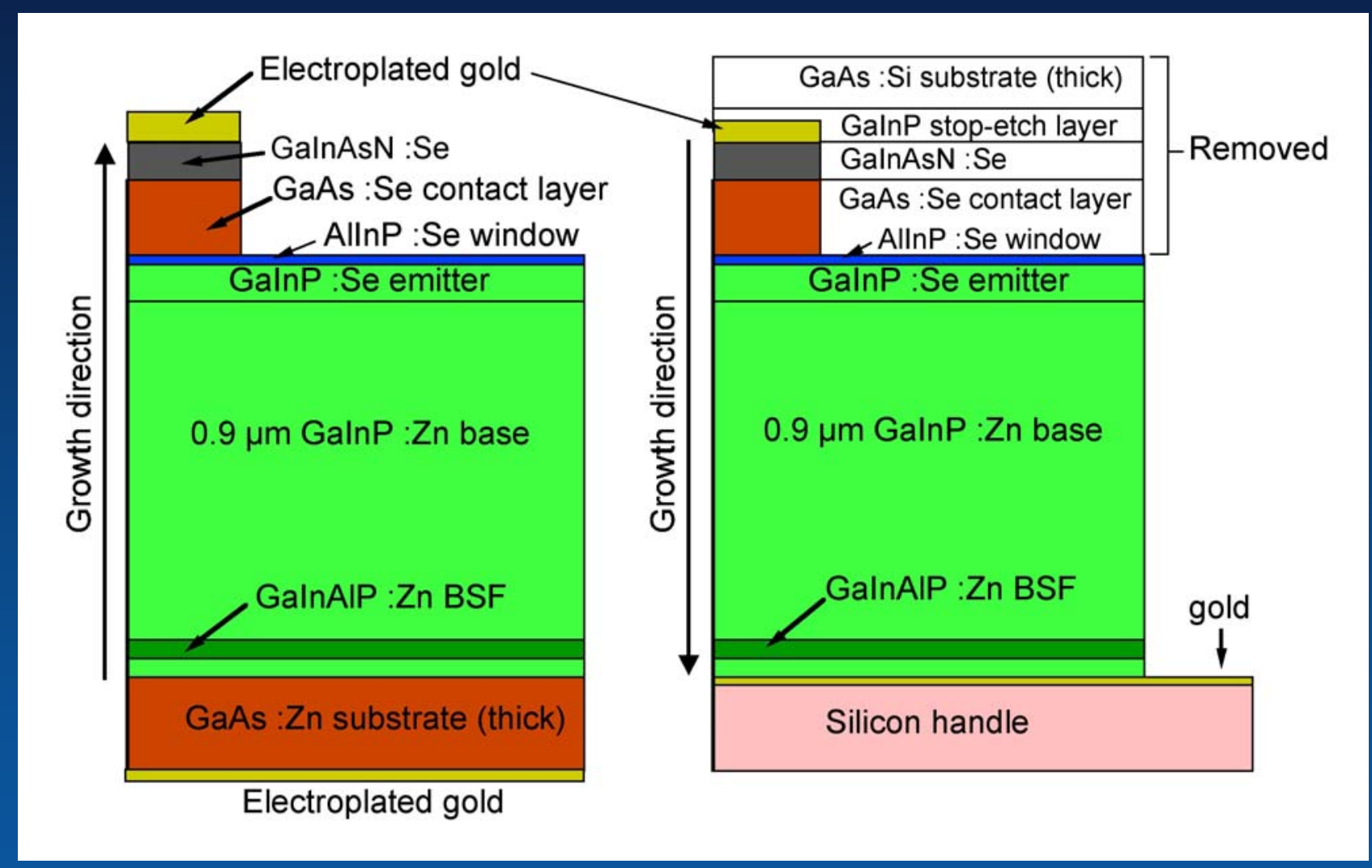

OMPVE

Atmospheric pressure

Vertical reactor

Precursors:

Trimethylgallium

Triethylgallium

Trimethlyindium

Trimethlyaluminum

Arsine

Phosphine

Dimethylhydrazine

Diethylzinc

Hydrogen selenide 


\section{Solar cell characteristics (optimized for inverted growth)}

\begin{tabular}{|c|c|c|c|c|c|c|c|c|c|}
\hline & $\begin{array}{c}\mathrm{R}_{\mathrm{s}} \\
(\Omega / \mathrm{sqr})\end{array}$ & $\begin{array}{c}\mathrm{R}_{\mathrm{c}} \\
\left(\mathrm{m} \Omega-\mathrm{cm}^{2}\right)\end{array}$ & $\begin{array}{c}\text { Emitter } \\
\left(10^{18} \mathrm{~cm}^{-3}\right)\end{array}$ & $\begin{array}{c}\text { Base } \\
\left(10^{16} \mathrm{~cm}^{-3}\right)\end{array}$ & $\begin{array}{c}\text { Voc } \\
(\mathrm{V})\end{array}$ & $\begin{array}{c}\text { Jsc } \\
\left(\mathrm{mA} / \mathrm{cm}^{2}\right)\end{array}$ & $\begin{array}{c}\mathrm{FF} \\
(\%)\end{array}$ & $\begin{array}{c}\text { Eff } \\
(\%)\end{array}$ \\
\hline 1A & inv & 326 & 0.088 & -3.2 & 1.4 & 1.395 & 10.97 & 88.3 & 13.5 \\
\hline 1B & up & 981 & 0.11 & -0.98 & 0.34 & 1.372 & 10.79 & 86.8 & 12.9 \\
\hline
\end{tabular}

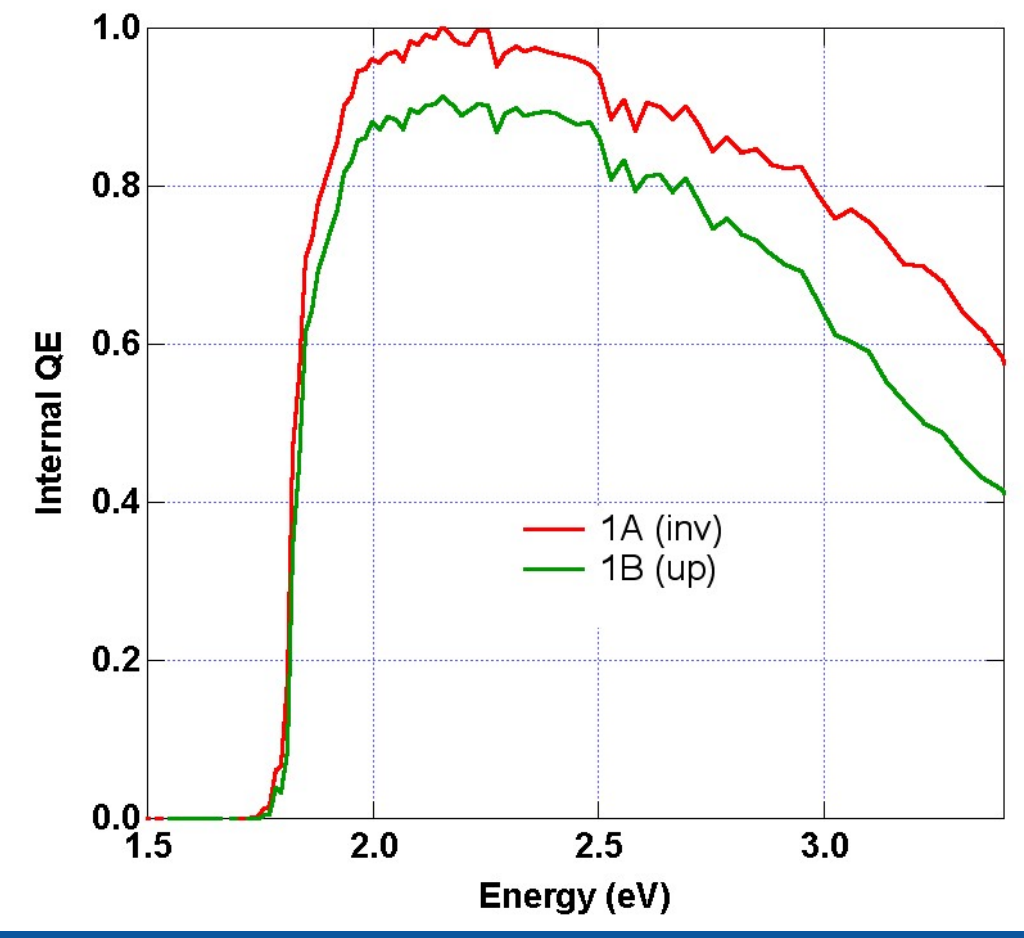

GalnAsN contacts for $1 \mathrm{~A}$ and $1 \mathrm{~B}$

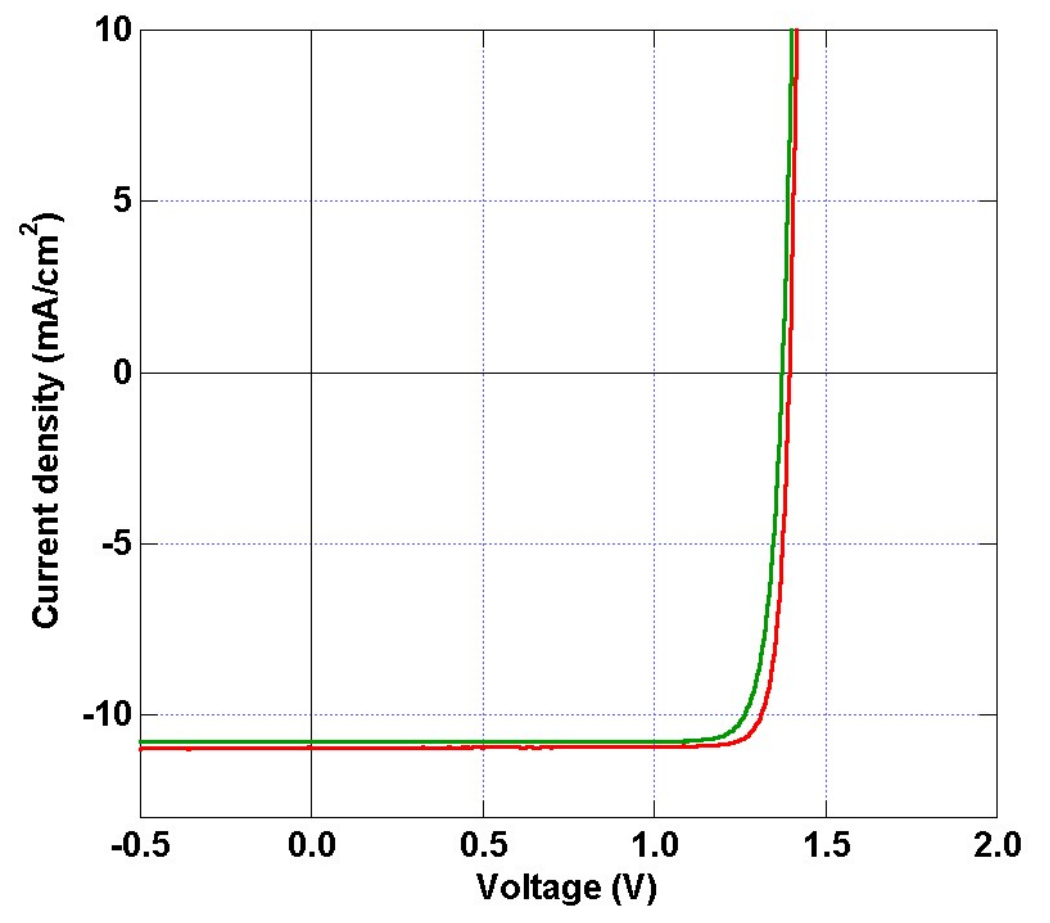

XT-10

GalnP reference cell calibrated for lowAOD 


\section{Solar cell characteristics (optimized for upright growth)}

\begin{tabular}{|c|c|c|c|c|c|c|c|c|c|}
\hline & $\begin{array}{c}\mathrm{R}_{\mathrm{s}} \\
(\Omega / \mathrm{sqr})\end{array}$ & $\begin{array}{c}\mathrm{R}_{\mathrm{c}} \\
\left(\mathrm{m} \Omega-\mathrm{cm}^{2}\right)\end{array}$ & $\begin{array}{c}\text { Emitter } \\
\left(10^{18} \mathrm{~cm}^{-3}\right)\end{array}$ & $\begin{array}{c}\text { Base } \\
\left(10^{16} \mathrm{~cm}^{-3}\right)\end{array}$ & $\begin{array}{c}\text { Voc } \\
(\mathrm{V})\end{array}$ & $\begin{array}{c}\text { Jsc } \\
\left(\mathrm{mA} / \mathrm{cm}^{2}\right)\end{array}$ & $\begin{array}{c}\mathrm{FF} \\
(\%)\end{array}$ & $\begin{array}{c}\text { Eff } \\
(\%)\end{array}$ \\
\hline 1C & up & 600 & 0.50 & -1.7 & 9.1 & 1.361 & 11.27 & 88.6 & 13.6 \\
\hline $1 \mathrm{D}$ & inv & -- & -- & -- & 4.5 & 1.306 & 11.31 & 68.7 & 10.2 \\
\hline 1E & inv & 516 & 0.22 & -2.1 & 3.1 & 1.340 & 11.03 & 86.3 & 12.8 \\
\hline
\end{tabular}
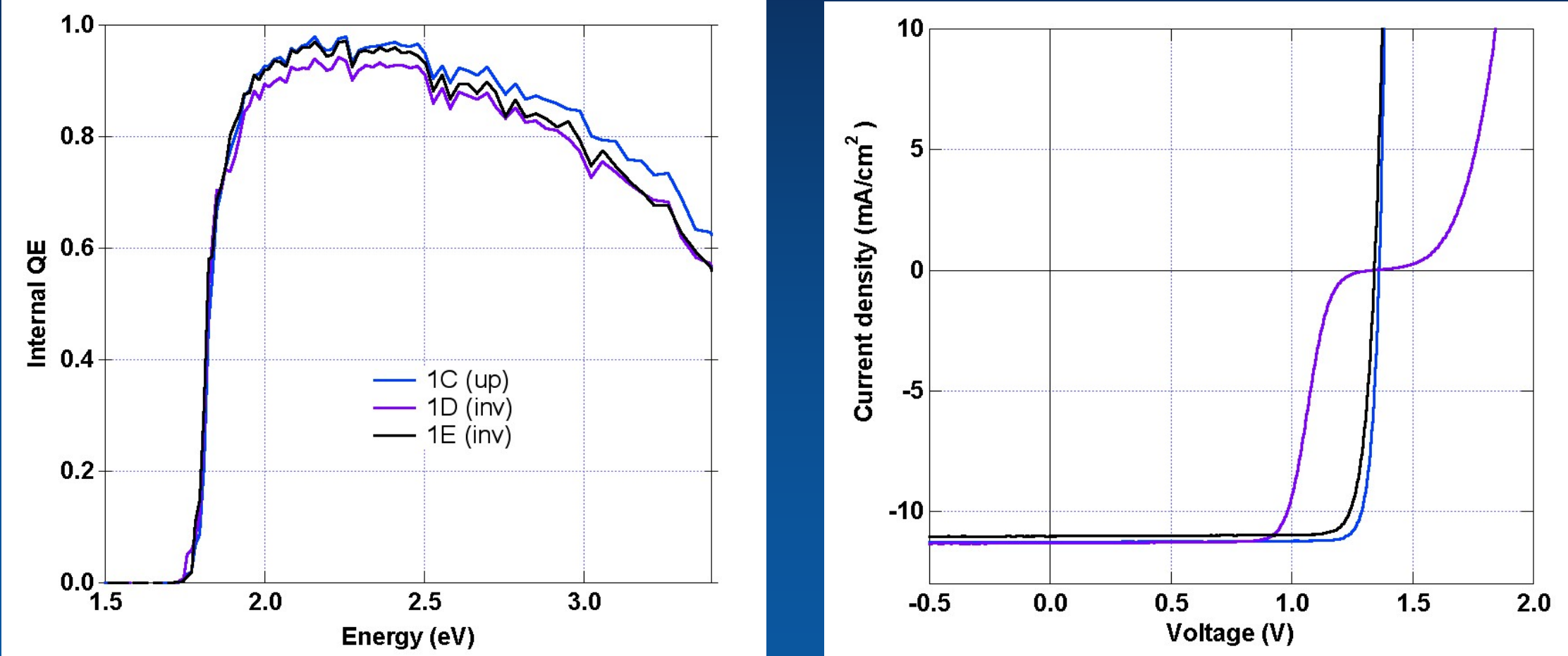

GaAs contacts for $1 \mathrm{C}$ and $1 \mathrm{D}$; GalnAsN contacts for $1 \mathrm{E}$ 


\section{SIMS on inverted-upright pair (1A-1B)}

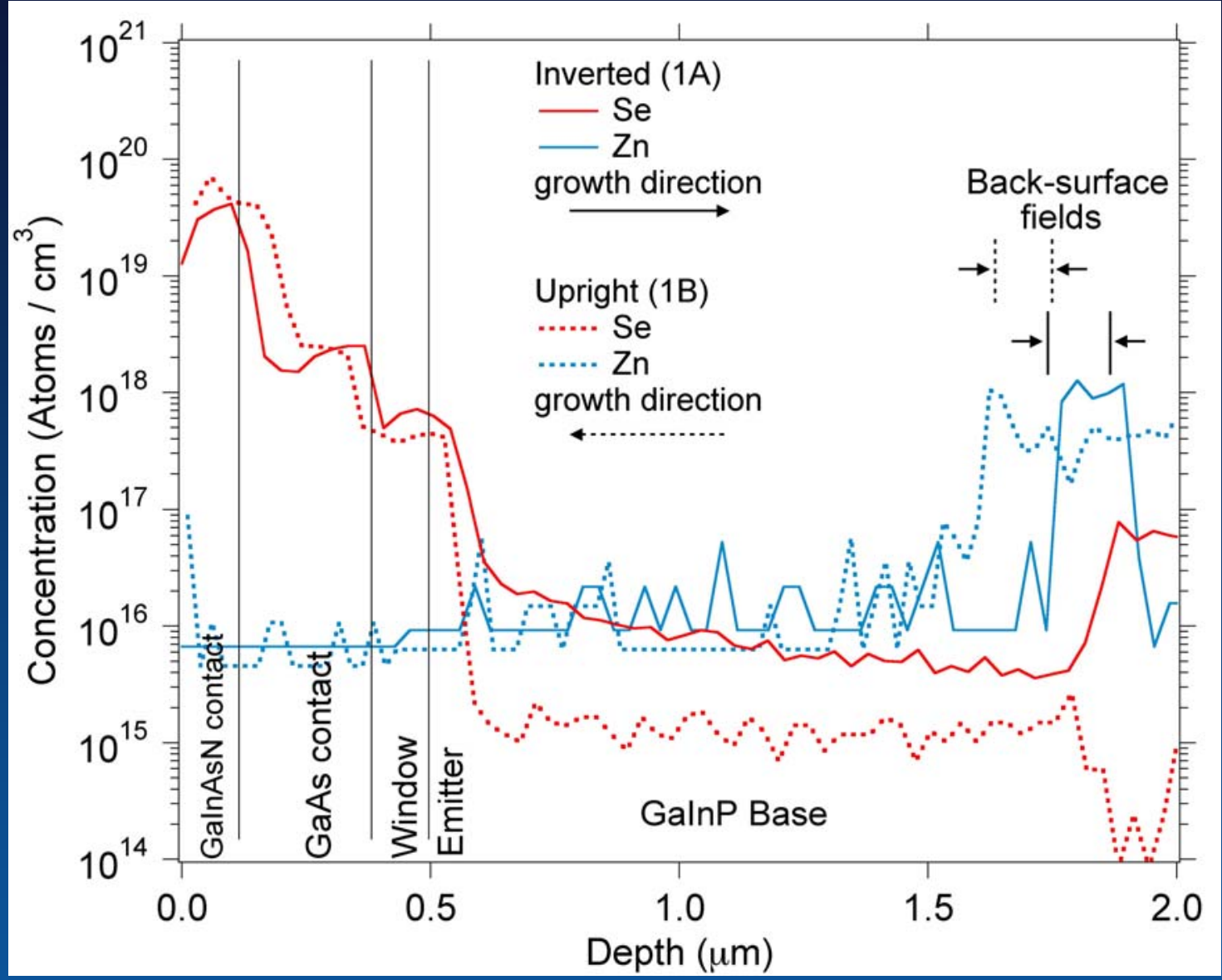

Junction depth

Selenium tails

Zinc tails at BSF

Window layer doping

Contact layer doping

Cell thickness

\begin{tabular}{|c|c|c|c|c|c|c|c|c|c|}
\hline & $\begin{array}{c}\mathrm{R}_{\mathrm{s}} \\
(\Omega / \mathrm{sqr})\end{array}$ & $\begin{array}{c}\mathrm{R}_{\mathrm{c}} \\
\left(\mathrm{m} \Omega-\mathrm{cm}^{2}\right)\end{array}$ & $\begin{array}{c}\text { Emitter } \\
\left(10^{18} \mathrm{~cm}^{-3}\right)\end{array}$ & $\begin{array}{c}\text { Base } \\
\left(10^{16} \mathrm{~cm}^{-3}\right)\end{array}$ & $\begin{array}{c}\text { Voc } \\
(\mathrm{V})\end{array}$ & $\begin{array}{c}\text { Jsc } \\
\left(\mathrm{mA} / \mathrm{cm}^{2}\right)\end{array}$ & $\begin{array}{c}\mathrm{FF} \\
(\%)\end{array}$ & $\begin{array}{c}\text { Eff } \\
(\%)\end{array}$ \\
\hline 1A & inv & 326 & 0.088 & -3.2 & 1.4 & 1.395 & 10.97 & 88.3 & 13.5 \\
\hline 1B & up & 981 & 0.11 & -0.98 & 0.34 & 1.372 & 10.79 & 86.8 & 12.9 \\
\hline
\end{tabular}




\section{SIMS, cont'd (arsenic)}

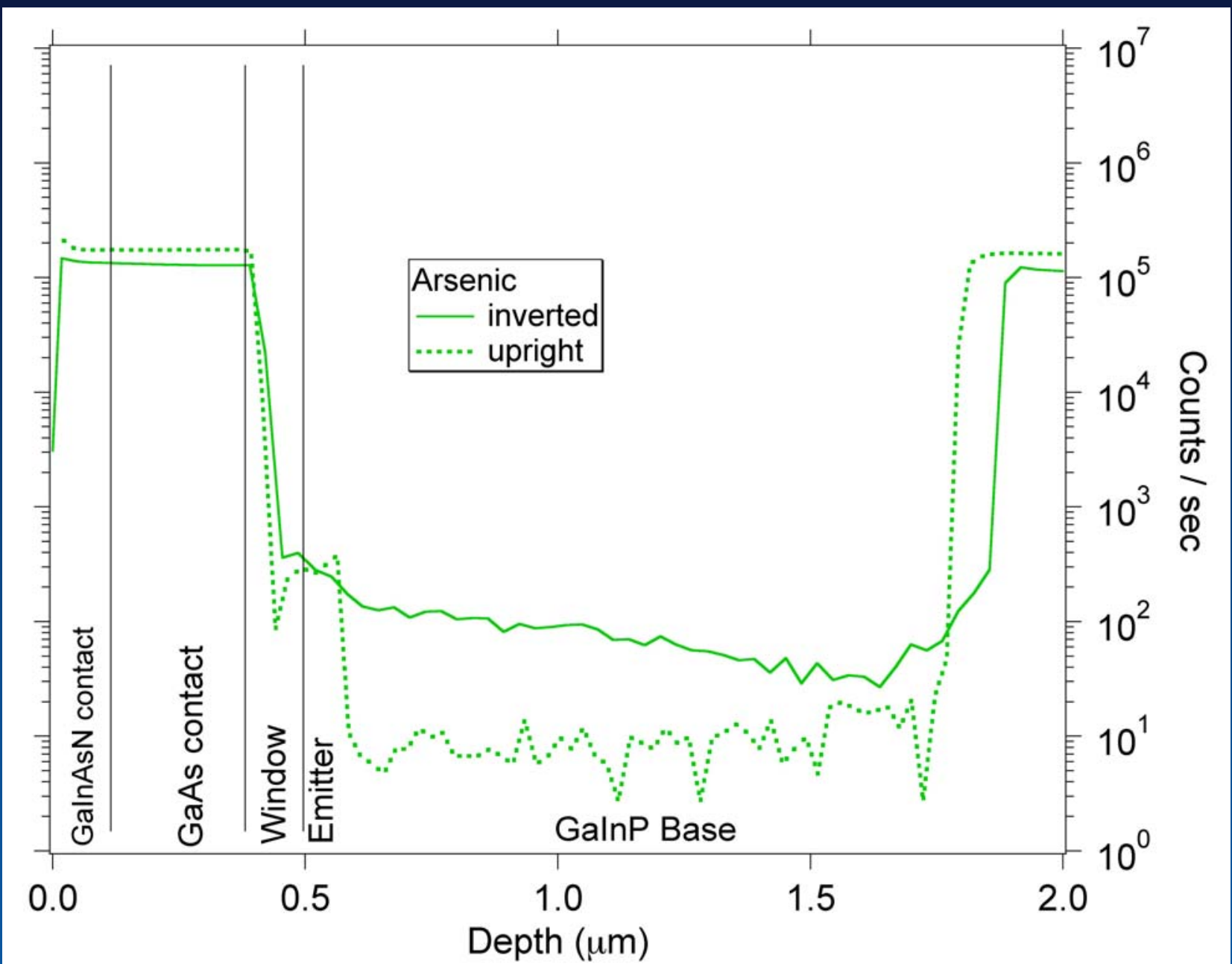

Long arsenic tail in the inverted cell

$\rightarrow$ memory effect in the growth reactor. 


\section{Effect of the top contact layer}

$\mathrm{JV}$ curves for all inverted growths

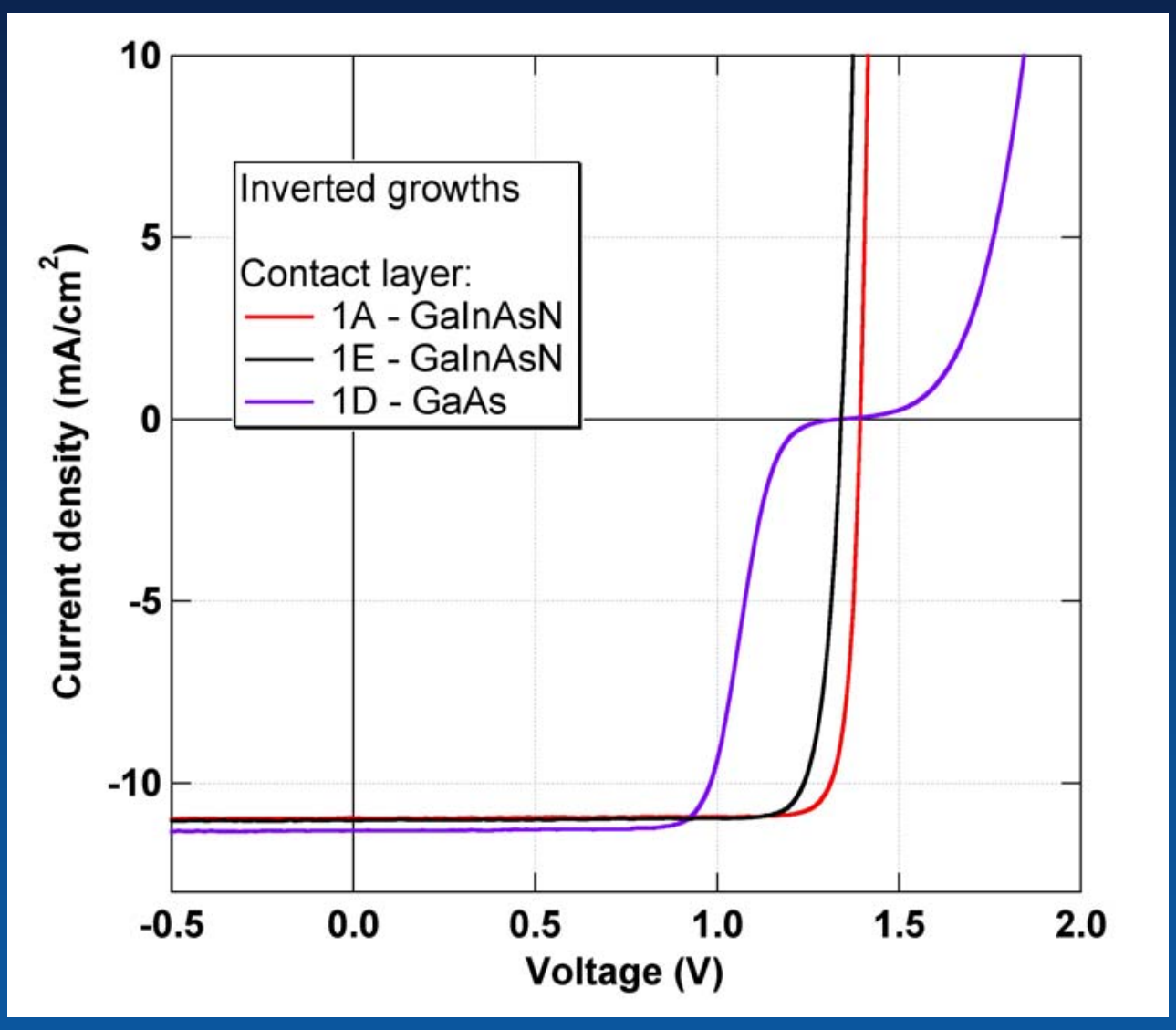

Introducing $\mathrm{N}$ has been found to:

(1) lower the bandgap (GalnAsN with 1\% N has $\mathrm{E}_{\mathrm{g}} \sim 1.1 \mathrm{eV}$ )

$\longrightarrow$ Lower contact resistance

(2) increase the effective mass

$\longrightarrow$ Higher carrier concentration We speculate that it will also:

(3) inhibit diffusion 


\section{Diffusion in GalnAsN}

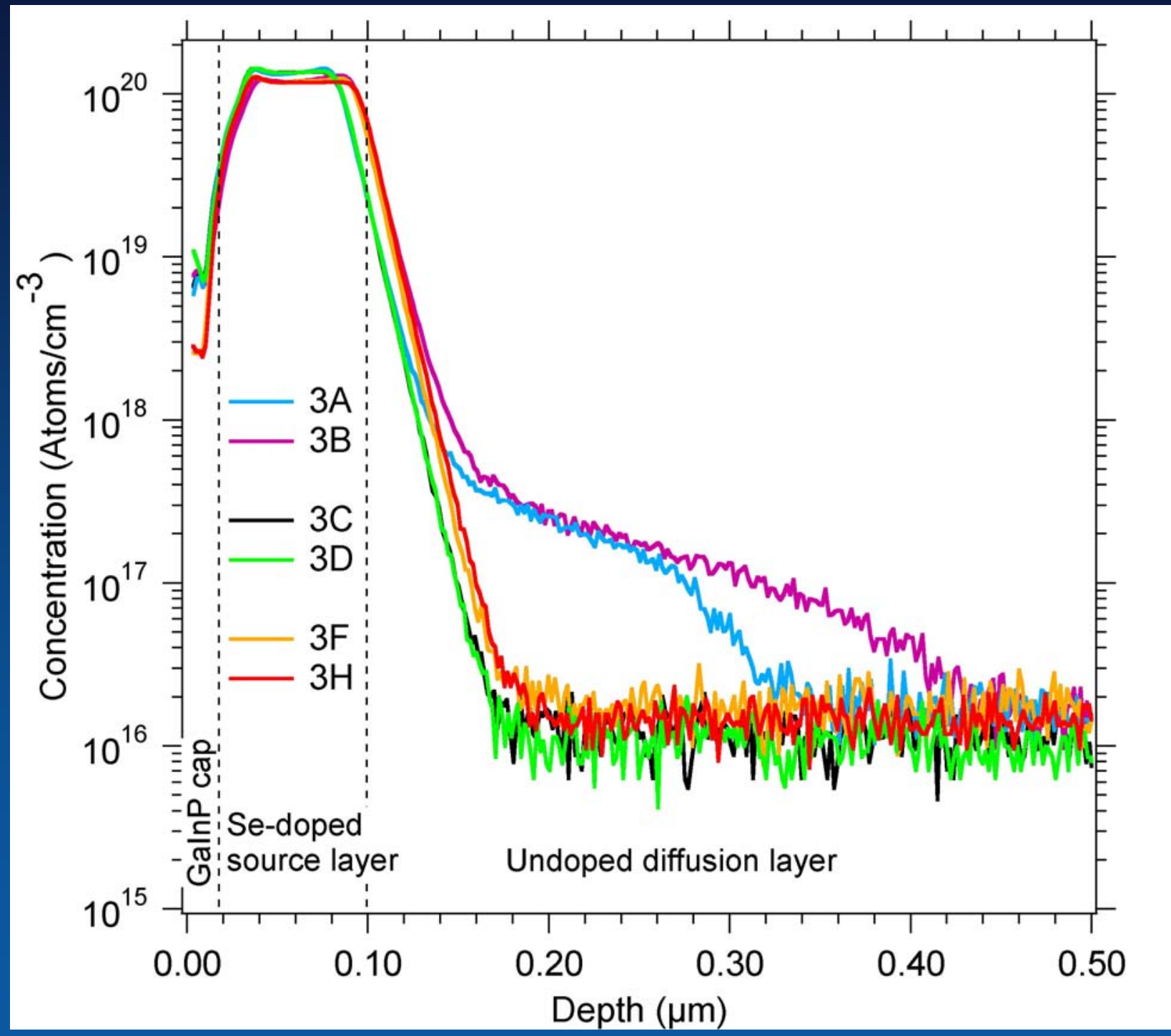

$\mathrm{PH}_{3}$ overpressure @ $650^{\circ} \mathrm{C}$

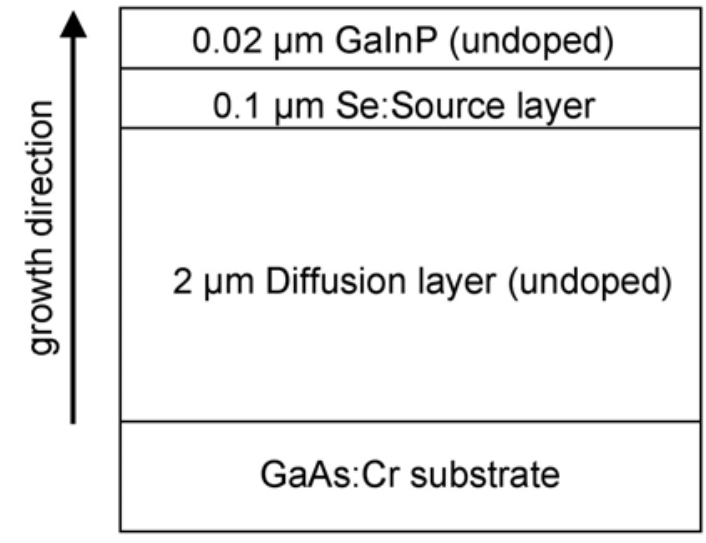

\begin{tabular}{|l|l|}
\hline 3A & $\begin{array}{l}\text { Se:GaAs / GaAs } \\
\text { (not annealed) }\end{array}$ \\
\hline 3B & Se:GaAs / GaAs \\
\hline 3C & $\begin{array}{l}\text { Se:GaAs / GalnAsN } \\
\text { (not annealed) }\end{array}$ \\
\hline 3D & Se:GaAs / GalnAsN \\
\hline 3F & $\begin{array}{l}\text { Se:GalnAsN / } \\
\text { GalnAsN }\end{array}$ \\
\hline 3H & Se:GalnAsN / GaAs \\
\hline
\end{tabular}

NREI National Renewable Energy Laboratory 


\section{Contact resistance study}

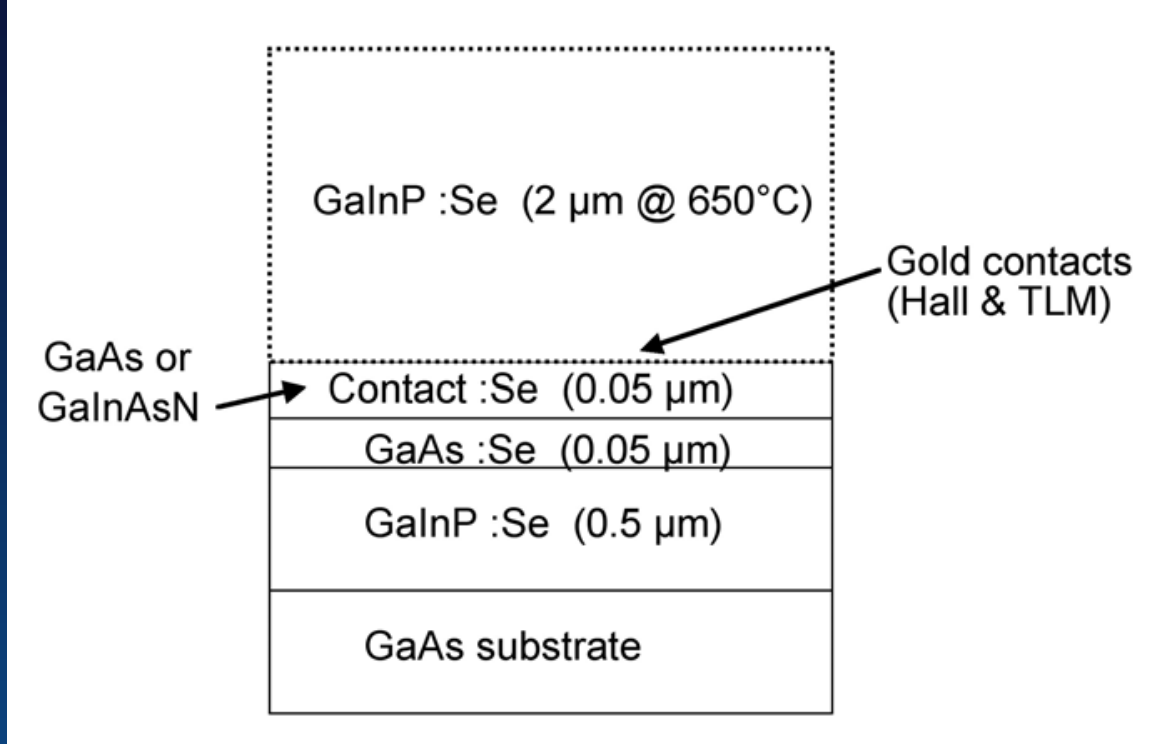

GalnAsN composition:

$\sim 1 \% \mathrm{~N}$

$2 \%$ In for latticematching to $\mathrm{GaAs}$

Contact layer grown at $570^{\circ} \mathrm{C}$

\begin{tabular}{|c|l|c|}
\hline Sample & \multicolumn{1}{|c|}{ Contact layer } & $\begin{array}{c}\text { Specific contact resistance } \\
\left(\mathrm{m} \Omega-\mathrm{cm}^{2}\right)\end{array}$ \\
\hline 2A & GaAs (not annealed) & 0.013 \\
\hline 2B & GaAs (annealed) & 1.98 \\
\hline 2C & GalnAsN (not annealed) & 0.003 \\
\hline 2D & GalnAsN (annealed) & 0.020 \\
\hline
\end{tabular}




\section{Summary}

Excellent performance is achievable in both upright and inverted configurations with proper consideration.

Subtle differences in depth profile, QE and JV between upright and inverted growths due to dopant diffusion.

GalnAsN contact layer is resilient to lengthy annealing; more work necessary to determine why. 


\title{
Acknowledgements
}

Michelle Young

Waldo Olavarria

Charlene Kramer

\author{
III-V group at NREL
}

This work was supported by the U.S. Department of Energy under Contract No. DE-AC36-99G010337 with the National Renewable Energy Laboratory.

myles_steiner@nrel.gov

(303) 384-7675 